\title{
Integration of a New Column-Parallel ADC Technology on CMOS Image Sensor
}

\author{
Fan Z. Nelson \\ Electrical and Computer Engineering \\ University of Idaho, \\ Moscow, Idaho, U.S.A \\ fan.nelson@vandals.uidaho.edu
}

\author{
Suat U. Ay \\ Electrical and Computer Engineering \\ University of Idaho, \\ Moscow, Idaho, U.S.A \\ suatay@uidaho.edu
}

\begin{abstract}
A new analog-to-digital converter (ADC) technology called Single-slope look ahead ramp (SSLAR) analog-to-digital converter (ADC) was proposed for column-parallel CMOS image sensors. Additionally, a corresponding programmable ramp generator for SSLAR ADC was also designed in such a way that it only allows flexible code hopping (between 0 and 127 least significant bit (LSB)), code fall back and look-ahead operations in column-parallel ramp ADC. This new ADC technology is able to provide conversion speed improvement depending on individual image information with less than $1.0 \%$ image quality degradation. Simulation demonstrated that the conversion speed of this new ADC technology is 4-5x higher than a traditional single-slope ADC with minimal circuitry for processing 8-bit standard gray images as well as 3-4x for standard CIF videos. For processing higher resolution images, the conversion speed may further increase. A prototype chip using the 8-bit SSLAR ADC architecture was realized in a $0.5 \mu \mathrm{m}, 2 \mathrm{P3M}$, CMOS process with a layout area of $8.2 \mathrm{~mm}^{2}$.
\end{abstract}

Keywords- Analog to Digital Converter; ADC; single-slope look ahead ramp ADC; single-slope ramp ADC; integrating ADC.

\section{INTRODUCTION}

Currently, several different types of column parallel ADCs (Analog to Digital Converters) have been used in CMOS (Complementary Metal Oxide Semiconductor) image sensors. A 12-bit column parallel ADC with accelerated ramp [1] was published in 2005 and exploits a way to reduce the number of ramp steps based on the nature of photon shot noise limitation. The results show that the image quality is not affected by the accelerated ramp modulation when shot noise $\left(\mathrm{M}_{\text {shot }}\right)$ is equal or less than $0 \mathrm{~dB}$. Additionally, conversion speed can be greatly enhanced by reducing the number of ramp cycles without degradation of image quality.

The concept of "a new simultaneous multislope (SMS) analog-digital converter (ADC) architecture" [2] came out one year later. The proposed SMS ADC architecture works like a column parallel single-slope ADC except in that it has one comparison phase and one slope phase. Furthermore, several slopes are used in parallel during the slope phase, where each slope covers a part of the total signal swing. The result indicates that, when compared to the single-slope ramp (SSR) ADC, SMS ADC has a conversion speedup approximately 2 times and requires only a small amount of extra circuitry. The disadvantage is a relative low conversion speedup improvement.

An additional method of increasing conversion speed has been suggested by using a new column-parallel ADC architecture based on a multiple-ramp single-slope (MRSS) ADC [3, 4] which improves upon the techniques in [2]. Further increases in conversion speed can be made by combining the MRSS ADC architecture with the concept of exploiting the amplitude-dependent characteristic of photo shot noise present in image signals [1]. But the disadvantage here is that it is difficult to implement such a large number of ramps. First, each output of the ramp generator must be buffered in order to drive the capacitive load presented by a larger number of comparators. Since the number of comparators connected to each ramp is signal-dependent, each buffer has to be dimensioned for worst case situations, where it has to drive all of the comparators. Therefore, there is tradeoff between the increased speed obtained by using a large number of ramps and increased power dissipation of using many ramp buffers.

Two-step single-slope ADCs based on multiple ramp signals have been reported in [2] and [4]. However, these twostep single-slope ADCs require several ramp generators corresponding to the number of coarse ADC steps. This architecture has increased area and power consumption due to the multiple ramp generators and multiple ramp signal lines. Therefore, coarse ADC resolution is limited to only 2 or 3 with little speed improvement. A new two-step SS ADC using a single ramp generator for the coarse and fine ADCs has been proposed $[5,6]$. This technique is free from ramp slope mismatch and high power consumption that were the bottlenecks of the conventional two-step SS ADCs with multiple ramp generators. The results demonstrate that the proposed ADC has ten times higher conversion speed without any significant increase in power consumption when compared to the conventional SS ADC.

In contrast with the techniques mentioned above, our method uses an alternative approach which also combines the statistical analysis of the images. In this paper, we introduce a new ADC algorithm and column parallel ADC architecture integrated on a column-parallel CMOS image sensor to reduce 
SSR-ADC's latency without degrading the image quality and integrity. The new ADC introduces code hopping, fall back, and code look-ahead operations considering the statistics on the sampled row signals. We call this architecture a single slope look-ahead (SSLAR) ADC architecture. This paper focuses on the introduction of the SSLAR ADC algorithm and its implementation and simulation using MATLAB as well as AMIS $0.5 \mu \mathrm{m}$ process to verify the proper operations.

This paper is organized as follows. Section II introduces the topology of the SSLAR ADC. A corresponding programmable ramp generator topology is introduced in section III. Design and simulation results are reported in section IV. Section V is dedicated for conclusion.

\section{SingLE-SLOPE LOOK-AHEAD RAMP (SSLAR) ADC}

In order to address the speed issue for single slope ramp (SSR) ADC, a new ADC algorithm and topology called single-slope look-ahead ramp (SSLAR-ADC) ADC was reported [7]. Correspondingly, a programmable ramp generator with changeable code hopping was also introduced. The new ADC algorithm was successfully implemented in MATLAB. Simulation on both standard gray images and CIF videos were conducted. It was determined that, by setting proper threshold and jump step, the algorithm results in increased ADC speed; improving frame rate of the CMOS image sensor without greatly degrading image quality [7]. For a single input, conversion can be greatly improved by using SSLAR ADC when a proper step size is chosen. For example, when a step size of 24 is chosen a speedup of approximately $6 \mathrm{x}$ can be achieved when compared to SSR ADC (refer to Fig.1-2). An additional 8-bit standard sample image "LENA" can also be observed in Fig.3.

Different jump steps were considered during simulation to plot both mean-square error (MSE) rated in percent of full scale and conversion speed up. It was determined that, for proper thresholds, jump step sizes between 4 and 6 LSB achieve an ADC speedup approximately $4-5 \mathrm{x}$ times and results in less than $1 \%$ image quality degradation for 8 -bit standard gray image. In this case, jump step sizes between 4 and 6 can be claimed as an ideal jump step size range if $256 \times 256$ (8-bit) standard gray images need to be processed. For processing higher resolution images, the thresholds may also be increased little. In the same fashion, for proper thresholds, larger jump step sizes could be set for processing higher resolution images. In conclusion, changeable step sizes are necessary depending on individual image information. Thus, a fully-flexible programmable ramp generator with flexible code hopping is needed in order to fit this new SSLAR ADC algorithm well.

\section{PROGRAMMABLE RAMP GENERATOR DESIGN}

In this section, only the digital circuitry will be described. The new programmable step SSLAR ADC composed of three major units is shown in Fig.4. They are the event detector (ED), controller unit (CONT) and the ramp-count generator (RCG) blocks. Step word N[6:0] can be programmed between 0 to 127 LSB for different applications.

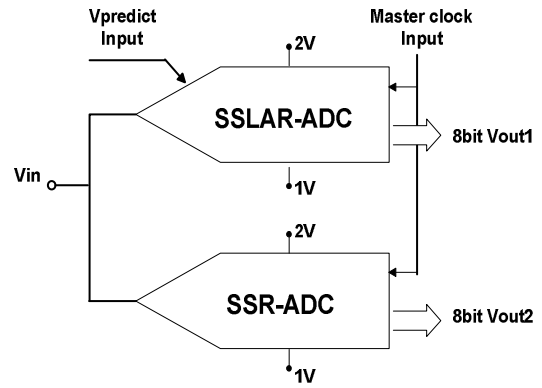

Figure 1. SSLAR-ADC Versus SSR-ADC with only one input.

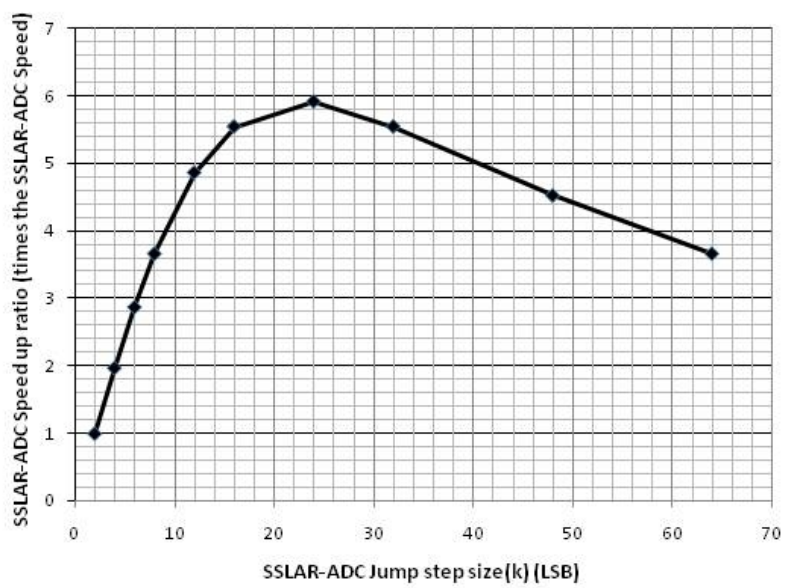

Figure 2. Speed up of the SSLAR-ADC compared to SSR-ADC with a single signal voltage input.
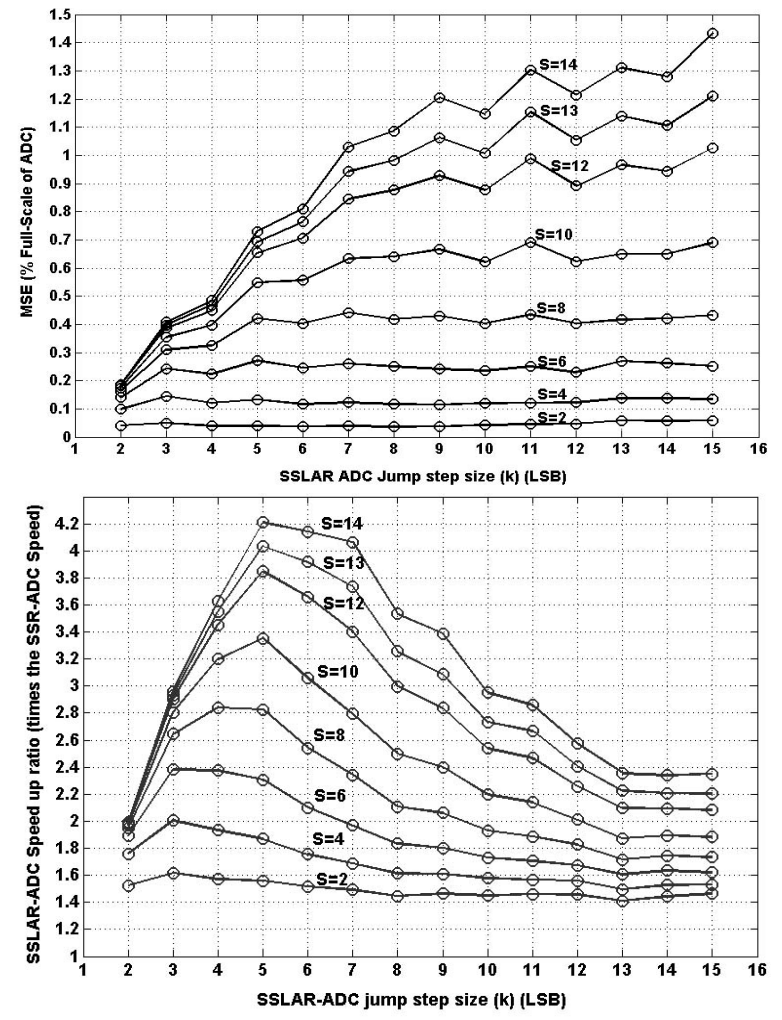

Figure 3. MSE versus jump steps for 8-bit "LENA" (upper figure) and ADC speed-up versus jump steps for 8-bit "LENA" (bottom figure). 


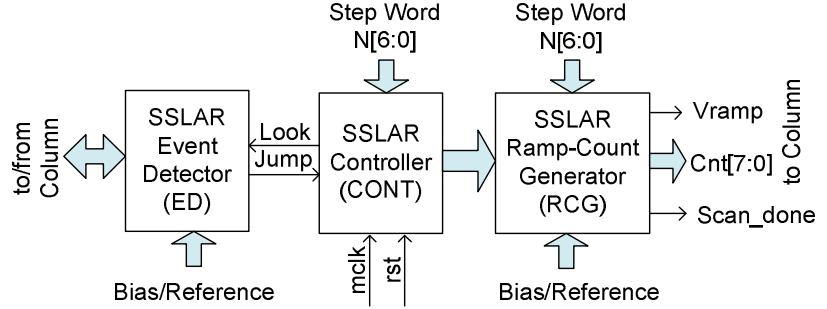

Figure 4. Programmable SSLAR ADC blocks.

\section{A. Event Detector (ED)}

The event detector (ED) is connected to the column predictor circuit and generates the "jump" signal depending on the "look" signal and column predictor conditions. ED also receives analog reference and bias signals.

\section{B. The Controller Unit (CONT)}

Controller unit (CONT) is central to the SSLAR ADC generates the "look" signal for the event detector (ED) and other control signal for ramp-count generator (RCG) blocks. CONT coordinates and implements the SSLAR ADC algorithm

A finite state machine (FSM) was used for generating six different operation states (refer to Fig.5 and Table.1) as well as internal and external control signals for CONT. Four control signals "rst" (external operation reset), "jump" (external jump signal from ED unit), "done" (internal counter done signal) and "mclk" (external master clock signal) determine the FSM states. FSM changes its state at rising edge of the master clock signal conditionally or unconditionally. At the same time, these six operation states also generate six additional control signals. Three of which are most important since they are externally fed to ED and RCG units. They are "look", "Lclk" and "C[2:0]" signals.

\section{SSLAR Ramp-Count Generator (RCG) Design}

The ramp-count generator (RCG) unit generates the analog ramp signal and the associated 8-bit digital counter output. Its block diagram is shown in Fig.6. RCG is composed of two multiplexers, one carry look-ahead (CLA) full-adder with latch (FAL) block, two carry look-ahead subtractors and one 8-bit binary weighted charge scaling ramp generator block. Thus, look ahead, jump and fall back operations are controlled through the proper timing of the blocks.

Table I. Function description of six states for CONT unit.

\begin{tabular}{|c|c|}
\hline State & Function Description \\
\hline S0 & $\begin{array}{c}\text { Count starts at zero and ramp voltage starts at original } \\
\text { point (original state). }\end{array}$ \\
\hline S1 & $\begin{array}{c}\text { Ramp voltage look-ahead k step size and counter look } \\
\text { ahead } \mathrm{k} / 2 \text { step size from original. }\end{array}$ \\
\hline S2 & $\begin{array}{c}\text { Ramp voltage maintains the same, but counter jump rest } \\
\mathrm{k} / 2 \text { step size. }\end{array}$ \\
\hline S3 & $\begin{array}{c}\text { Ramp voltage continues to look ahead k step size and } \\
\text { counter look-ahead } \mathrm{k} / 2 \text { step size from previous start point. }\end{array}$ \\
\hline S4 & $\begin{array}{c}\text { Ramp voltage falls back k step size and counter falls back } \\
\mathrm{k} / 2 \text { step size from previous start point due to unsuccessful } \\
\text { look ahead. }\end{array}$ \\
\hline S5 & Ramp voltage and counter raise one step size per time. \\
\hline
\end{tabular}

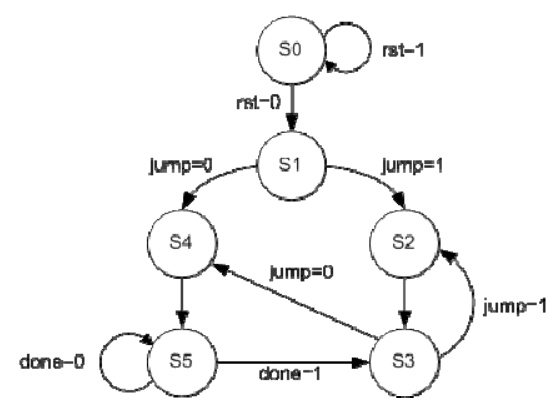

Figure 5. State machine diagram of the controller unit

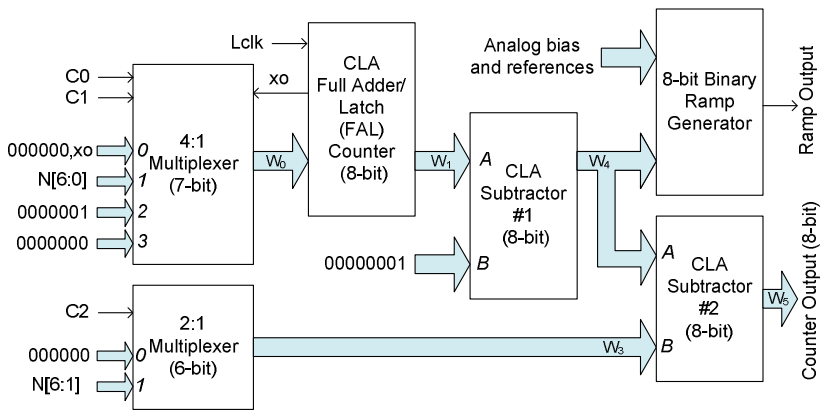

Figure 6. SSLAR ADC ramp-count generator unit block diagram

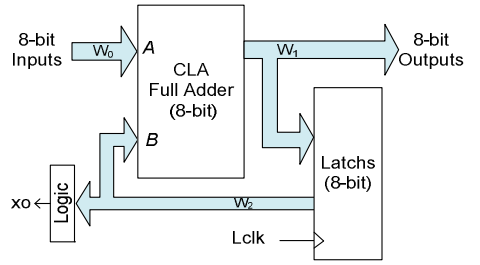

Figure 7. Block diagram of CLA full-adder and latch (FAL) unit

Block diagram CLA full-adder and latch (CLA-FAL) unit is the central part of the look-ahead and jump operation. It is shown in Fig.7. Latch clock (Lclk) is generated from CONT unit and fed to the RCG unit. One 8-bit input (A[7:0]) to CLA full-adder are provided by the $4: 1$ multiplexer unit

and another 8-bit input (B[7:0]) are provided by the 8-bit latch outputs. CLA full-adder adds these two inputs to generate the CLA-FAL block output and feedback to the 8-bit latch. When Lclk signal is asserted from low to high, latch block holds the outputs of the CLA-FAL unit.

Multiplexer selection code $\left(\mathrm{C}_{0}, \mathrm{C}_{1}, \mathrm{C}_{2}\right)$ are generated by the CONT unit based on the state of FSM. For 4-to-1 multiplexer, when $\mathrm{C}_{0}=$ ' 0 ' and $\mathrm{C}_{1}=$ ' 0 ', xo value is passed; when $\mathrm{C}_{0}=$ ' 0 ' and $\mathrm{C}_{1}=$ ' 1 ', digital one is passed; when $\mathrm{C}_{0}=$ ' 1 ' and $\mathrm{C}_{1}={ }^{\prime} 0$ ', the 7-bit step programming word $(\mathrm{N}[6: 0])$ is passed; input $\mathrm{C}_{0}=$ ' 1 ' and $\mathrm{C}_{1}=$ ' ' 1 ' was never selected and was set to zero. By taking upper 6 bit of the original step program word from 4-to-1 multiplexer, half step code word is attained from 2-to-1 multiplexer. 2-to-1 multiplexer always passes the half step programming words. When $\mathrm{C}_{2}=$ ' 1 ', it look ahead half step size; when $\mathrm{C}_{2}={ }^{\prime} 0$ ', it jump rest half step size and implements the full step size counter increment operation. An 8-bit binary weighted capacitive digital to analog converter was used as ramp generator. 


\section{LAYOUt DESIGN AND SimUlation RESUlts}

Proposed design was fabricated in a $0.5 \mu \mathrm{m} 2 \mathrm{P} 3 \mathrm{M}$ CMOS process. Supply voltage is $3.3 \mathrm{~V}$ and layout of 8 -bit ramp generator is shown in Fig.8. Core area SSLAR-ADC is approximately $0.54 \mathrm{~mm}^{2}$.

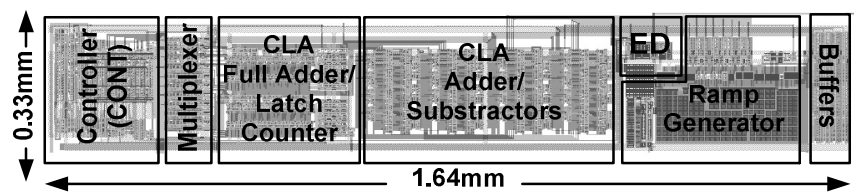

Figure 8. Layout of 8-bit programmable ramp generator.

Jump step size can be easily programmed between 0 and 127 LSB. Here, sample step size 8 was chosen and corresponding simulation results are shown in Fig.9. The jump or fall back operation consumes two clock cycles.

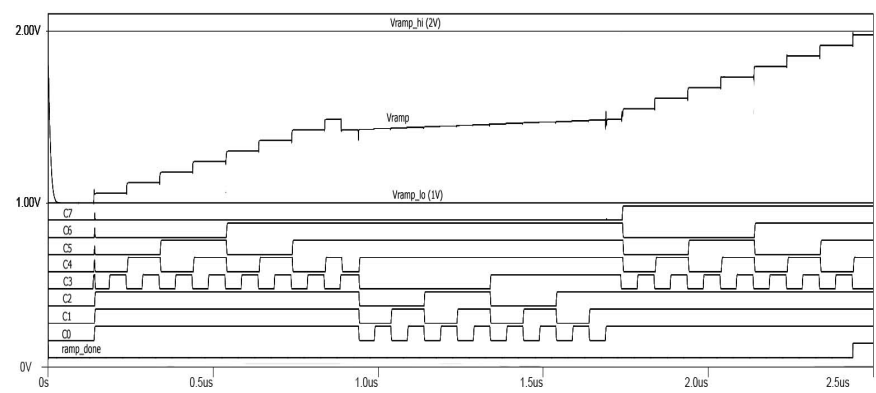

(a)

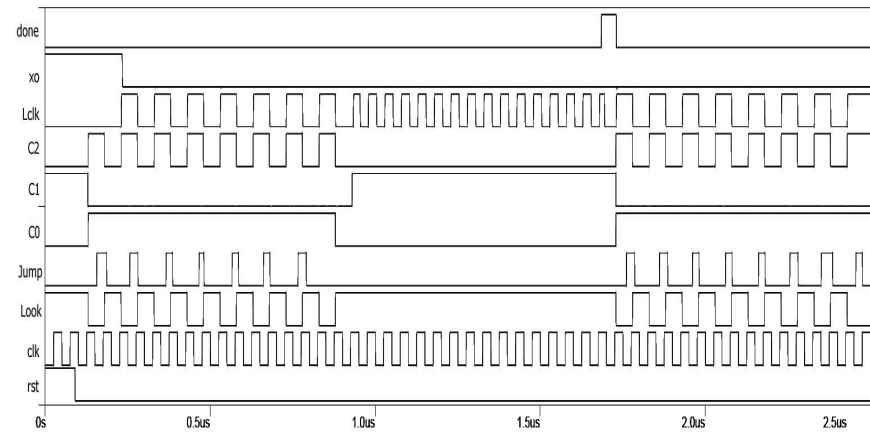

(b)

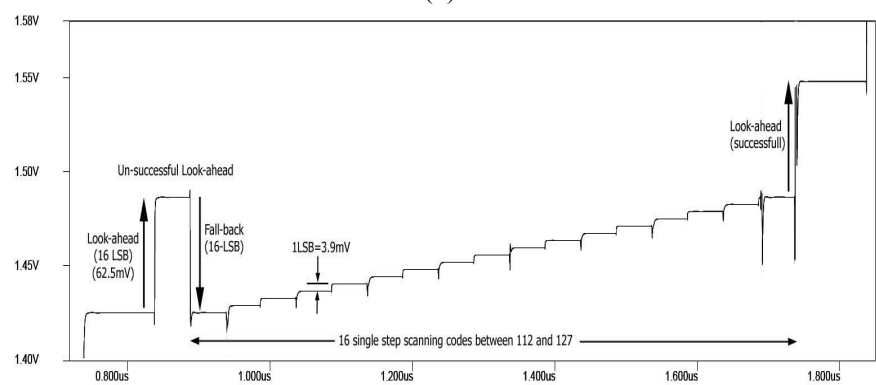

(c)

Figure 9. 8-bit full block simulation results of the SSLAR ADC's controller and ramp-count generator unit with step $=16 \mathrm{LSB}$, and jump signal is forced; (a) analog signals, (b) digital control signals (c) Zoomed ramp output voltage (Vramp) for failed look-ahead operation between codes 112 and 127.

\section{CONCLUSION}

Compared to the single slope ramp (SSR) ADC, the singleslope look-ahead ramp (SSLAR-ADC) ADC's conversion speed was greatly enhanced due to skipping of code ranges where few pixels in that range lie without image degradation. A 12-bit ADC architecture could be easily expanded based on the 8-bit architecture and would be expected to enhance conversion speed even more. A potential downside to SSLAR architecture is the increased power consumption with extra circuitry. Table II contains a comparison of other available methods to our proposed technique. The proposed programmable ramp generator can attain variable step sizes which can optimize the image process quality as well as conversion speed in a user specific manner. Future directions could be a combination of photon shot noise limitation techniques with our SSLAR-ADC design and may enhance our ADC's conversion speed even more.

\section{ACKNOWLEDGEMENT}

We would like to thank Micron Technology Foundation for support and MOSIS fabrication service for allowing proposed design to be fabricated in a subsidized $0.5 \mu \mathrm{m} 2 \mathrm{P} 3 \mathrm{M}$ CMOS run (PID\#82059).

Table II. Available methods

\begin{tabular}{|c|c|c|c|c|c|}
\hline $\begin{array}{c}\text { Ref } \\
\cdot\end{array}$ & $\begin{array}{c}\text { Speedup } \\
\text { (times) }\end{array}$ & $\begin{array}{c}\text { Die Area } \\
\left(\mathbf{m m}^{\mathbf{2}}\right)\end{array}$ & Year & $\begin{array}{c}\text { Process } \\
(\boldsymbol{\mu m})\end{array}$ & Power \\
\hline$[2]$ & $\sim 2$ & extra circuitry & 2006 & 0.35 & N/A \\
\hline$[4]$ & $\begin{array}{c}\text { MRSS: 3.3 } \\
\text { MRMS: 4.4 }\end{array}$ & $5 \times 5$ & 2007 & 0.25 & $52 \mathrm{~mW}$ \\
\hline$[6]$ & $\sim 10$ & $3.6 \times 3.2$ & 2009 & 0.35 & $36 \mathrm{~mW}$ \\
\hline$[7]$ & $\mathrm{BW}(8 \mathrm{~b}): \sim 5$ & $2.0 \times 4.1$ & 2009 & 0.5 & $56 \mathrm{~mW}$ \\
\hline
\end{tabular}

\section{REFERENCES}

[1] Toshinori Otaka et al., "12-bit Column-parallel ADC with accelerated ramp,'Institute of electronnics, Information and communication engineers, vol.105. no. 185, pp. 35-38, 2005.

[2] Leif Lindgren, "A new simulataneous Multislop ADC architecture for array implemetation," IEEE Trans. On Circuit Syst.II, Exp. Briefts, vol.53, no.9, pp.921-925, Sep,2006.

[3] Snoeij, et al., "A CMOS Image sensor with a column-level multipleramp single-slope ADC," ISSCC 2007, pp 506,618,11-15 Feb.2007.

[4] M. F. Snoeij, et al., "Multiple-ramp column-parallel ADC architectures for CMOS image sensors," IEEE J. Solid-State Circuit, vol.42, no.12, pp.2998-3006, Dec.2007.

[5] J. Lee,et al., "A 10b column-wise two-step single-slope ADC for highspeed CMOS image sensor," in Proc. IEEE Int. Image sensor Workshop, Ogunquit, ME, pp. 196-199, Jun. 2007.

[6] S.Lim,et al., " A high-speed CMOS Image sensor with column-parallel two-step single-slope ADCs" IEEE Trans. Electron Devices, vol.56,no.3, pp.393-398, Mar.2009.

[7] F. Z. Nelson, M. N. Alam, and S. U. Ay, "A Single-slope look-ahead ramp (SSLAR) ADC for column parallel CMOS Image sensors, ” IEEE Workshop on Microelectronics and Electron Devices, WMED 2009, 3 April 2008 